ISSN: 2277-3754

ISO 9001:2008 Certified

International Journal of Engineering and Innovative Technology (IJEIT)

Volume 10, Issue 10, April 2021

\title{
A Comparative Analysis of Fibre Bragg Grating Sensor and Conventional Electrical Strain Gauge Model for Structural Health Monitoring
}

ObajeS. P, Abdullahi Z. M,AgbonE. E\&OgweR

Department of Electronics and Telecommunications Engineering, Ahmadu Bello University, Zaria, Nigeria

\begin{abstract}
This research work presents a comparative analysis of Fibre Bragg Grating (FBG) sensor model and the conventional Electrical Strain Gauge (ESG) model for Structural Health Monitoring (SHM). SHM have been mainly deployed in telecommunication facilities owing to their exceptional ability to monitor infrastructures in hazardous environment. In this research, the FBG sensitivity was compared with that of the ESG sensor at different strain and stress values, at Poisson's ratio (v) of 0.19525 and 0.3 , at effective refractive index $\left(n_{\text {eff }}\right)$ of 1.44 and 1.5, and Gauge Factor $(G F)$ of 1.1 and 1.5 respectively. $F B G(A)$ is the FBG sensor with Poisson's ratio of 0.3 and effective refractive index of 1.44, while $F B G(B)$ is the $F B G$ sensor with Poisson's ratio of 0.19525 and effective refractive index of 1.5 . The simulation result shows that FBG(A)performs better than $F B G(B)$ by, $7.25 \%$ and $8.05 \%$ increment in sensitivity strain and stress. Also, $2.7 \%$ increment in sensitivity for ESG was achieved for induced strain and stress values on the composite structure at GF of 1.1 and 1.5 respectively. Additionally, it was observed from the obtained results that there exists a linear relationship between the FBG and ESG sensors. This observation shows that the FBG sensor can perfectly replace the conventional ESG sensor in $S H M$, in view of the additional advantages of the FBG sensor which are small size, immune to electromagnetic interference, light weight just to mention a few over ESG sensor.
\end{abstract}

Index Term- FBG, ESG, Poisson's ratio, Bragg wavelength, Sensitivity, SHM.

\section{INTRODUCTION}

Structural Health Monitoring (SHM) have been mainly deployed in telecommunication, civil facilities, medical, aircraft, oil and gas, sport and spacecraft industry. Owing to their exceptional ability to monitor infrastructures in hazardous environment. To implement SHM sensors are deployed on these structures for regular observation of these structures subjected to physical parameters for health monitoring. In this work conventional ESG and FBG sensors are presented [3].

\section{A. Conventional Electrical Strain Gauge}

ESG is a sensor used to measure engineering strain as shown in Figure (1).ESGs are deployed in many industrial sectors for monitoring the condition of structures mostly deployed in harsh environment [8]. It consists of a lead, conductive grid, which changes its electrical resistance when subjected to deformation. The deformation is caused by physical quantities from the environment in which the sensor is mounted as shown in Figure (2).

Manuscript received: 26 March 2021

Manuscript received in revised form: 24 April 2021

Manuscript accepted: 10 May 2021

Manuscript Available online: 15 May 2021

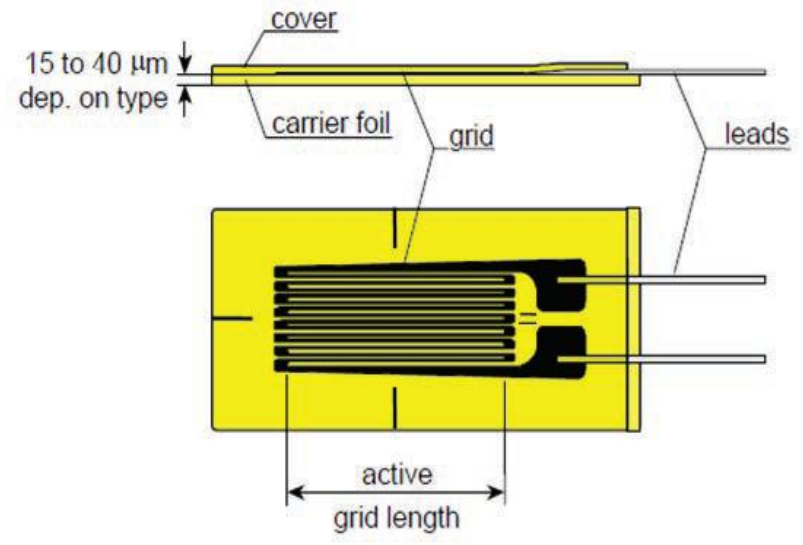

Fig.1. ESG [8]

The strain on the specimen is directly transferred to the gauge, generating a change in its resistance, the change in resistance corresponding to the physical change of the structure under investigation [9]. The conventional strain gauge is powered by an electrical source, to sense the physical quantity, the data gets to the data acquisition system, they are analyzed then the result is displayed as output as shown in the block diagram in Figure (3);

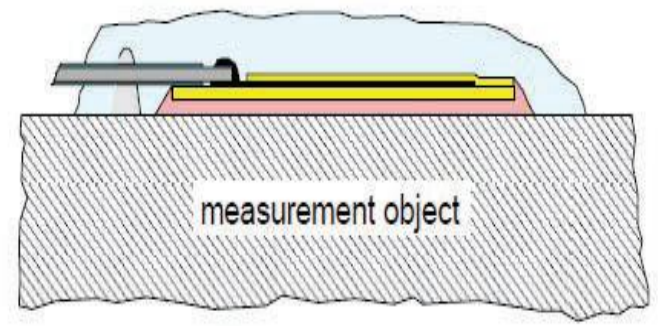

strain gage $\square$ adhesive $\square$ cover

Fig.2. Mounted ESG on a Structure [8]

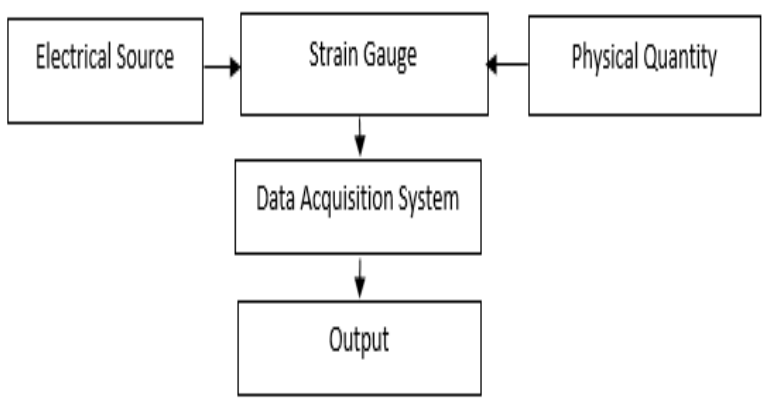

Fig.3. Block Diagram of an ESG System 
ISSN: 2277-3754

\section{ISO 9001:2008 Certified \\ International Journal of Engineering and Innovative Technology (IJEIT) \\ Volume 10, Issue 10, April 2021}

\section{B. Fibre Bragg Grating Based Sensing}

FBG sensing technology possesses a good multiplexing capability which enable it to accommodate many grading and different sensing like; strain, temperature, pressure, vibration, displacement, deformation just to mention a few in a fibre optic cable [7]. A fibre Gittering Bragg is a form of light reflecting and transmitting all others in the core of optical fibres, making it possible to utilise the lighting sensor [6]. The centre of the fibre will be covered by a silica covering that is less refractive than the core with a diameter from $4 \mu \mathrm{m}$ to $9 \mu \mathrm{m}$, which allows the wavelength inside the fibre to be formed [6], as seen in the figure (4).

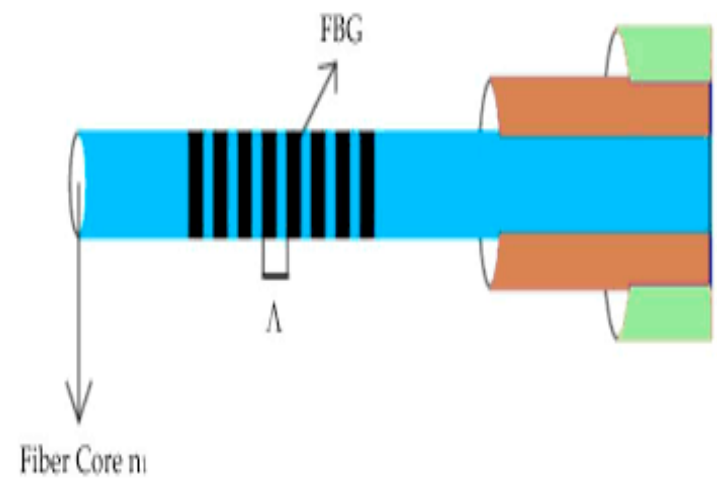

Fig. 4. FBG Sensor [10]

\section{RESEARCH OBJECTIVES}

The research objective is focused on carrying out comparative analysis of Fibre Bragg Grating (FBG) sensor model and the conventional Electrical Strain Gauge (ESG) model for Structural Health Monitoring (SHM). This analysis will help to showcase the properties of both FBG and ESG.

\section{OVERVIEW}

In this research a comparative analysis of Fibre Bragg Grating sensor and the conventional Electrical Strain Gauge model was carried out. The small size of FBG gave FBG sensors an edge over ESG sensor in the area of size, light weight, less complexity and immune to electromagnetic interference [1]. It is imperative that these sensors with its attendant advantages can serve as a replacement for the convention ESG sensors. The FBG sensitivity was compared with that of the ESG sensor at different strain and stress values, at Poisson's ratio of 0.19525 and 0.3 , at effective refractive index of 1.44 and 1.5, and Gauge Factor of 1.1 and 1.5 respectively. FBG (A), is the FBG sensor with Poisson's ratio of 0.3 and effective refractive index of 1.44. While,FBG (B) is the FBG sensor with Poisson's ratio of 0.19525 and effective refractive index of 1.5. The sensitivity of ESG and the FBGs were compared to observe the relationship between the sensors.

\section{LITERATURE REVIEW}

[11] Proposed an application of fibre Bragg grating in a real-time casing damage monitoring work. In the work a distributed FBG sensors analytical method was carried out to compare the main type and damage casing.
[12] Developed a distributed strain sensing using optical fibre sensing technology. Also, in the research, dynamic and distributed as regards with the fatigue loading was quantified using the technique known as Brillion Optical Correlation Domain Analysis (BOCDA).

[12] Proposed a technique of delay and sum technique for the confinement of multi-damage on large complex structures. The technique was structured based on the transmission properties of lamp waves as the pre-processing of defect localization. The localization precision of the delay and sum technique were meaningfully impacted by the uncertainty displayed by the velocity of the wave. The boundary processing method was intended to expand the delay and sum algorithm for the multi-damage case. Two parameters are actualized which are firmly identified with the health status of the structure. The primary parameter was the damage index of actuator sensor, which uncovered the contrast among measured response signal and baseline signal of a particular actuator sensor path. The second parameter was the damage profile of the area which signifies the possibility of damage presence in the subarea. In the monitoring procedure, the relating response signal (estimated signal) is compared with the baseline signals. The damage was considered as the dispersing source in a lamb wavebased structure health monitoring technique. Which captures the damage induced scattering signals accurately on the structure. The dispersive nature of the structure limits the coverage distance of the propagating signals which makes it difficult to quantify large area in structural health monitoring.

Proposed a method used to identify the significant damages of composite materials in aviation structure. In particular, impairment to skins, stringers and frames, utilizing ultrasound guided wave differential technique. The statistical processing was used for the estimations for normalize the test for the ordinary pattern of the test structure. In order to maximize the likelihood of discovery and minimizing the probability of false alarms. The nondestructive evolution approach depends on the waveguide nature of the structure, and uses carefully chosen guided wave modes and statistical analysis to distinguish defects on the composite structure. The contact approach (for laboratory development) and the non-contact approach (increasingly appropriate for field use) was created and tried on boards with now damage. The inspection execution of these procedures has been surveyed by obtaining the Receiver Operating Characteristic curves that have demonstrated incredible possibility for discovery of skin and stringer damage on the structure. This technique involved the deployment of many connecting cables, and consumed a lot of time during installation process. This makes the application of the technique very expensive.

\section{FBG SENSOR MODEL}

FBG sensors are defined with a unique wavelength that changes with induced physical parameters [6]. The fibre 
ISSN: 2277-3754

\section{ISO 9001:2008 Certified \\ International Journal of Engineering and Innovative Technology (IJEIT) \\ Volume 10, Issue 10, April 2021}

refractive aircraft Bragg gratings are termed Bragg planes which are made by constructively interfering with two opposing light sources [2]. The FBG deletion causes the sensor index of refraction to shift [6] as seen in Figure (5).

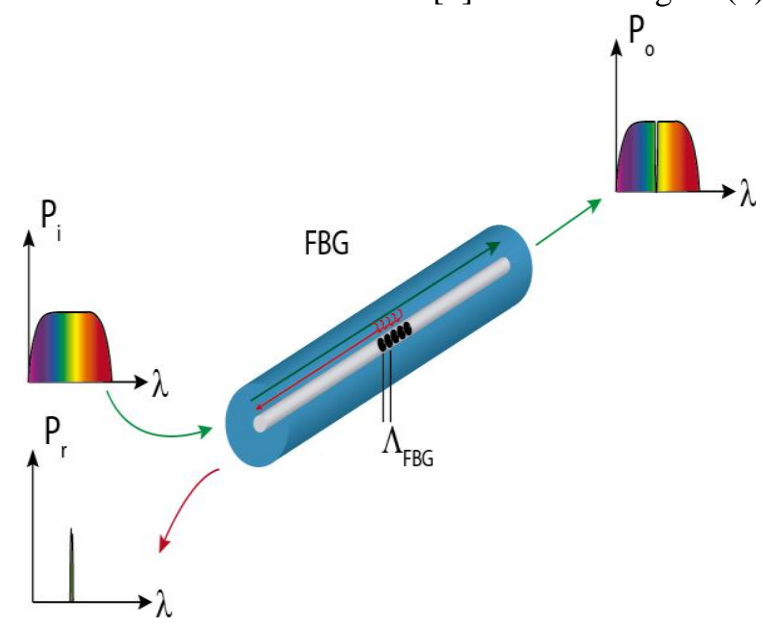

Fig.5. FBG Working Principle [2]

The grating wavelength is expressed by equation (1) [6]:

$$
\lambda_{B}=2 n_{\text {eff }} \Lambda
$$

Shift in Bragg wavelength of an FBG sensor $(\Delta \lambda)$ due to engineering strain is calculated by the formula given in equation (2) [3]

$$
\Delta \lambda=\Delta \lambda_{f b g 1}-\Delta \lambda_{f b g}
$$

where $\Delta \lambda_{f b g}$ is the wavelength shift of FBG1 due to strain and temperature, while $\Delta \lambda_{f b g z}$ is the wavelength shift of FBG2 due to temperature [3], the shift in FBG1 is expressed in equation (3) [10]

$$
\Delta \lambda_{f b g}=\lambda_{B}\left(\alpha_{f}+\xi\right) \Delta T+\lambda_{B}\left(1-P_{\theta}\right) \Delta \varepsilon(3)
$$

where $\alpha_{f}$ is the thermal expansion co-efficient of the FBG sensor, $\xi$ is the thermal-optic coefficient of the FBG sensor and $P_{\varepsilon}$ is effective strain optic coefficient. The change in FBG2 due to change in temperature is expressed in equation (4) $[11]:$

$$
\Delta \lambda_{f b g 2}=\lambda_{B}\left(\alpha_{f}+\xi\right) \Delta T(4)
$$

where $\lambda_{B}$ is the Bragg wavelength of FBG2 sensor and $\Delta T$ is the change in temperature, while equation (5) is the expression for the sensitivity of FBG sensor due change in strain [1]:

$$
\left.\Delta \lambda_{B}=\lambda_{B}\left[1-P_{B}\right]\right) \Delta \varepsilon
$$

The expression for the strain optics coefficient is shown in equation (6) [1]:

$$
P_{\theta}=\frac{n_{2 f f}^{2}}{2}\left\{p_{12}-v\left[\left(p_{11}+p_{12}\right)\right]\right\}(6)
$$

Where $v$ is the Poisson's ratio and $\mathrm{p}_{11}, \mathrm{p}_{12}$ are the Pockel's coefficient of the strain-optic tensor [1].

\section{CONVENTIONAL ESG MODEL}

The model of an Electrical Strain Gauge (ESG) of resistance of a wire with uniform cross section area $(A)$ and resistivity $(\rho)$ can be expressed in equation (7) [4]:

$$
R=\rho \frac{L}{A}(7)
$$

If the gauge is made from a length of uniform wire and subjected to a change in length, the resistance of the wire is expressed in equation (8) [4]:

$$
\Delta R=\rho \frac{L}{A}-(\rho+\Delta \rho) \frac{L+\Delta L}{A+\Delta A}(8)
$$

For a wire subjected to engineering strain, where $\Delta L<<L$ and $\Delta A<<A$. and $\Delta \rho<<\rho$. Where $\Delta$ is the change in the quantity, then the expression can be simplified by approximating $\Delta$ with the differential change, $d[4]$ :

$$
\begin{aligned}
& \Delta R \cong d R=\Delta\left(\rho \frac{L}{A}\right)(9) \\
& \frac{d R}{R}=\frac{d \rho}{\rho}+\frac{d L}{L}-\frac{d A}{A}(10)
\end{aligned}
$$

The basic proportionality expression between resistance, strain and the change in resistance per unit resistance $(\Delta R / R)$ are shown in equation (11) [4]:

$\frac{d R}{R}=\frac{d \rho}{\rho}+(1+2 v) \varepsilon(11)$

Where $v$ is the Poisson's ratio and equation (12) shows the Gauge Factor (GF) of the strain gauge which is the measure of the sensitivity of the material as [4]:

$$
G F=\frac{\alpha R f_{R}}{\varepsilon}(12)
$$

From equation (11) the Gage Factor (GF) can be determined as [4]:

$$
G F=1+2 v+\frac{d \rho_{\rho}}{\varepsilon}(13)
$$

Where $v$ is the Poisson's ratio, $\varepsilon$ is the strain, ${ }^{d \rho} / \rho$ is the deferential expression for the change in resistance per unit resistance and Figure (6) is the flow chart of the research work.

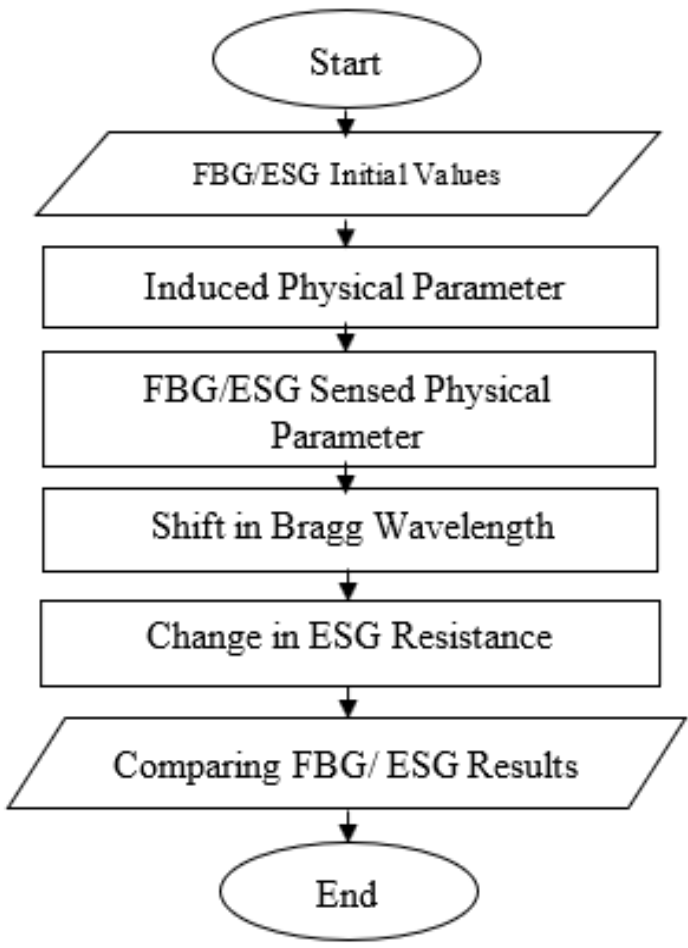

Fig.6. Flow Chart of the Proposed Work 
ISSN: 2277-3754

ISO 9001:2008 Certified

International Journal of Engineering and Innovative Technology (IJEIT)

Volume 10, Issue 10, April 2021

\section{SIMULATION RESULTS}

The results obtained from the simulation are discussed in this section. The following results are based on the FBG and ESG sensitivity to induced strain and stress on the composite structure.

FBG and ESG Sensitivity to Induced Strain and Stress on the Composite Structure

In Figure 7 and 8, the sensitivity of fibre Bragg grating sensors was plotted against that of the conventional electrical strain gauge. The plot was obtained, from the values in Table 1 and 2 during the simulation. Equations (5) and (12) was used to obtain the values in Table 1 and 2 when the GF is 1.1 and 1.5, Poisson's ratio is 0.19525 , and 0.3 , and $n_{\text {eff }}$ is at 1.44 and 1.5 respectively. From the plot, it can be observed that the $\operatorname{FBG}(\mathrm{A})$ model with the highest value result in higher sensitivity due to the increment in vand reduction in $n_{\text {eff }}$. Also, the ESG model with highest GF result higher sensitivity. The simulation result shows that the FBG sensor with the highest Poisson's ratio result in $7.25 \%, 8.05 \%$ increment in sensitivity to strain and stress when $n_{\text {eff }}$ is constant. Also, an increment of $2.7 \%$ in sensitivity for ESG with respect to GF of 1.1 and 1.5 was achieved.

Table 1: FBG and ESG Sensitivity to Strain

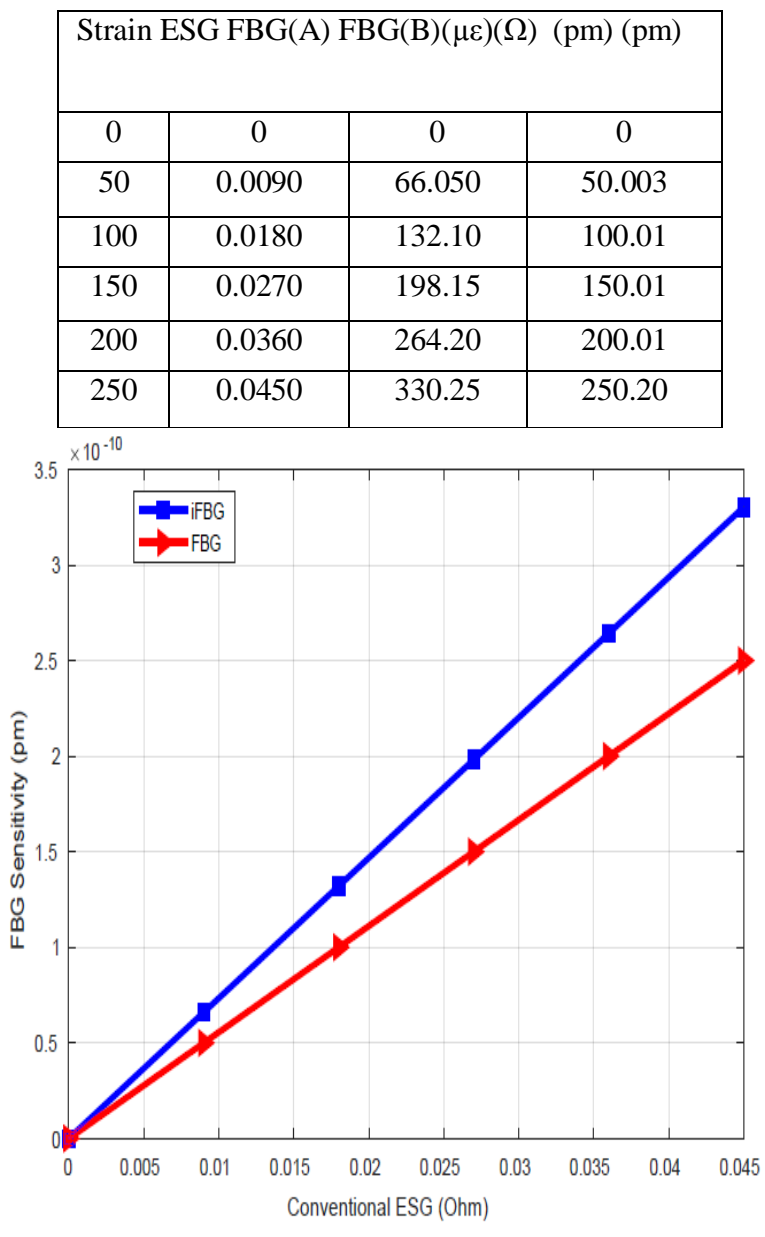

Fig.7. FBG verses ESG at GF of 1.5when the Composite Structure is Induced by Strain
Table 2. FBG and ESG Sensitivity to Stress

\begin{tabular}{|c|c|c|c|}
\hline \multicolumn{3}{|l|}{ Stress ESGFBG(A)FBG(B) (MPa) } & $(\Omega)(\mathrm{pm})(\mathrm{pm})$ \\
\hline 0 & 0 & 0 & 0 \\
\hline 6.750 & 0.0072 & 66.050 & 50.003 \\
\hline 13.50 & 0.0144 & 132.10 & 100.01 \\
\hline 20.25 & 0.0216 & 198.15 & 150.01 \\
\hline 27.00 & 0.0288 & 264.20 & 200.01 \\
\hline 33.75 & 0.0360 & 330.25 & 250.20 \\
& & & \\
& & & \\
\end{tabular}

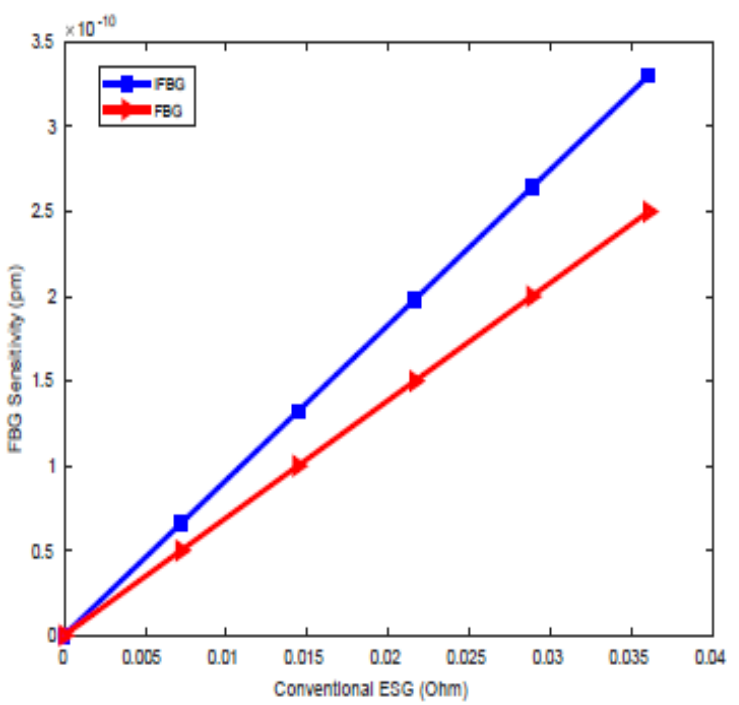

Fig.8.FBG verses ESG at GF of 1.1 when the Composite Structure is Subjected to Stress

VIII. CONCLUSION

In this research, a comparative study of FBG and conventional ESG model was presented. The FBG sensor with the highest value of Poisson's ratio result in $7.25 \%$, $8.05 \%$ increment in sensitivity to strain and stress. Also, $2.7 \%$ increment in sensitivity for ESG with respect to GF of 1.1 and 1.5 was achieved. From the analysis it is observed that FBG and ESG has a linear result with make the FBG sensitivity compactable with that of the ESG sensor. These makes FBG suitable for replace the ESG in sensing application.

\section{RECOMMENDATIONS}

The following are the recommended future works:

1. A research can be carried out to improve on the performance of FBG sensors.

2. The effect of increased sensitivity of ESG sensors can be studied with respect to it application in $5 \mathrm{G}$ technology.

\section{REFERENCES}

[1] Carlo, E.C., Antonello, C., Clarissa, C.A.Y., Vittorio, M. N. P., "Fibre Bragg Grating Based Strain Sensors: Review of Technology and Applications", Photonics Research Group. Sensors, PP: 1-27, 2018. 
ISSN: 2277-3754

ISO 9001:2008 Certified

International Journal of Engineering and Innovative Technology (IJEIT)

Volume 10, Issue 10, April 2021

[2] Garcia, I., Zubia, J., Durana, G., Aldabaldetreku, G. A., María A I., Joel V., "Optical Fibre Sensors for Aircraft Structural Health Monitoring", Sensors, 15, PP: 15494-15519, 2015.

[3] Jayant, K., Devendra, C., "FBG Based Strain Sensor with Temperature Compensation for Structural Health Monitoring", 4th Int'l Conf. on Recent Advances in Information Technology, India, PP: 1-4, 2018.

[4] Matthew, J. N., Rani, W. S., Lance, W., R., "Large Scale Applications Using FBG Sensors: Determination of in-Flight Loads and Shape of a Composite Aircraft Wing", NASA Engineering and Safety Centre., PP: 5-6, 2016.

[5] Mishra, P., Charan, K.B., Sethi, H.K., "Development of FBG Opti-wave system soft wear for structural health monitoring", International Journal of Engineering Science Invention, PP: 157-163, 2017.

[6] Prasad, U., Adane, D.S., "Application of Strain Gauges in Measurements of Strain Distribution in Complex Objects", Journal of Applied Computer Science and Mathematics JACSM, 6(2), PP: 135 -145, 2014.

[7] Satish, V. G. Nilaj, N. D., "Effect of Excitation Voltage and Lead Wire Resistance on Strain Measurement", 2nd International Conference for Convergence in Technology (I2CT), India, PP: 747-752, 2016.

[8] Capriottia, M. H. E. Kim, Lanza, S.F., Kim, H., "Detection of major impact damage to composite aerospace structures by ultrasonic guided waves and statistical signal processing", International Conference on Structural Dynamics, Volume 199, 2017, PP:1550-1555, 2017.

[9] Yi, P., Chong, H., Dandan, L. Shuangchun, Y., "The Application of Fibre Bragg Grating in the Real-time Casing Damage Monitoring Work", International Journal of Engineering Research and Development, Volume 4, Issue 6, PP: 40-44, 2012.

[10] Lamberti, A., Luyckx, G., Paepegem, W.V., Rezayat, A., Vanlanduit, S., "Detection, Localization and Quantification of Impact Events on a Stiffened Composite Panel with Embedded Fibre Bragg Grating Sensor Networks", .Sensors, 17, PP: 1-13, 2017.

[11] Shan, Shengbo, Li Cheng, and Fuzhen Wen., "Characterization of nonplanar second harmonic Lamb waves with a refined nonlinear parameter", Journal of Nondestructive Evaluation, Diagnostics and Prognostics of Engineering Systems, Vol 1, no. 1,PP:1-12, 2018.

[12] Capriotti, M., H. E. Kim, F. Lanza Scalea, and H. Kim., "Detection of major impact damage to composite aerospace structures by ultrasonic guided waves and statistical signal processing”, Procedia engineering, PP: 1550-1555, 2017. 